\title{
Gilbert NicOLAS
}

\section{Entre un espace muséologique et muséographique. Étude de cas : le Musée Archéologique de I'Université Saint-Esprit - Kaslik}

\section{Résumé}

À travers un travail expographique visant l'intégration du musée archéologique de I'Université Saint-Esprit Kaslik, au Liban, dans les enjeux contemporains qui traversent la vie estudiantine, cette étude s'intéresse à l'espace muséal et son importance sensorielle et communicationnelle au sein des musées universitaires. La recherche se concentre sur la distinction entre un espace muséologique et un espace muséographique, par la conception d'un nouvel espace architectural qui s'engage dans le processus communicationnel universitaire. Cet article et travail expographique résulte de l'importance des différents éléments qui constituent les espaces extérieur et intérieur des musées.

Mots-clés : architecture, musée, muséographie, espace, université.

\begin{abstract}
The expographic work in this research aims to integrate the archeological museum of the Holy Spirit University Kaslik, in Lebanon, into the contemporary issues introduced to students during their studies, and emphasizes the importance of museum space on a sensorial and on a communicational level within university museums. Through the design of a new architectural space that engages in the university communication process, the research focuses on the distinction between a museological space and a museographic space. This article and expographic work, together, shed the light on the importance of the different elements that make up the outer and inner spaces of museums.
\end{abstract}

Keywords : architecture, museum, museography, space, university. 


\section{Introduction}

L'architecture n'est pas seulement perçue visuellement, elle est également "visitée » et "utilisée » et, en tant que telle, devient partie intégrante, acteur physique d'une représentation où des sens, autres que la vision, sont impliqués (LETESSON 2009, p. 21).

Dans son discours volumétrique, l'architecte cherche à communiquer à l'utilisateur un espace qui le complète et qui incarne une extension physique de son corps. Cependant, le travail architectural consiste également à répondre, de la manière la plus claire, à la demande des utilisateurs pour rendre leurs expériences de l'espace cohérentes et adéquates. L'espace semble s'effacer autour du visiteur pour laisser place à la fonction voulue et attendue. De ce fait, l'étude de l'espace des bâtiments assure que le visiteur doit se trouver au centre, l'espace négatif et positif ${ }^{1}$ se modifiant pour son confort mental et physique, comme l'a précisé Jean-Pierre Laurent, au musée dauphinois de Grenoble, en 1976. L'espace appartient au public et l'incite à s'approprier la revitalisation de ce qui l'entoure.

Chaque type de bâtiment est spécifique de par son entité, ses détails, sa fonction et la finalité pour laquelle il a été créé. Sachant que les musées sont des établissements qui oscillent entre espace privé, semi-public et public, l'architecte vient travailler la volumétrie et l'espace intérieur en respectant simultanément la spécificité de chaque intervalle. L'étude complexe de la configuration spatiale et architecturale du musée influe directement sur l'expérience muséale et sur la pratique du visiteur, d'où la nécessité d'une approche qui déploie des moyens conséquents. Cette configuration spatiale entraîne ainsi le visiteur dans un évènement social, créant des relations complexes entre périodes, espaces et objets exposés, incitant l'esprit à la réflexion, à la curiosité, pour atteindre des apprentissages nouveaux. Dès la création du musée, l'espace architectural joue un grand rôle sur l'évolution des relations entre musée et public, et nous pouvons observer cet entrecroisement trilogique entre architecture, communication et inclusion socio-culturelle. Ainsi, l'architecture connote la destruction physique des barrières, incitant le visiteur à franchir les portes des bâtiments et à entrer dans ce dialogue matériel avec l'exposition et la collection. M. Stawiarski définit le seuil comme le lieu d'accueil et de passage; il est arrêt et franchissement, clôture et ouverture (STAWIARSKI 2010, p. 3). Une fois le seuil franchi, le visiteur se coupe du monde extérieur, il est « déprogrammé », déconnecté du quotidien, et plonge durant sa visite dans un monde nouveau (DAVALLON 2000, p. 174-175). Après cette transition, le visiteur entre dans la muséographie et dans le corps du musée, qui est son espace intérieur. L'étude de cet espace semble, dès la fin du $X X^{e}$ siècle, de plus en plus

\footnotetext{
${ }^{1}$ Espace positif et négatif se complètent, l'espace positif est l'espace occupé par une construction, un meuble ou un objet et l'espace négatif est défini par la surface vide qui entoure l'espace positif utilisée pour la circulation ou l'aération.
} 
important. Pour étudier l'espace du musée et de l'exposition, il est important de distinguer l'espace découlant du travail architectural de celui qui découle du travail expographique (GHARSALLAH-HIZEM 2008, p. 5).

Beaucoup de textes ont traité de la muséologie et de la muséographie ainsi que de leurs intersections dans le travail des musées et des expositions. Dans notre article, nous voulons mettre le point sur ces deux termes du point de vue architectural. Nous montrerons brièvement ce que nous entendons par la distinction entre les deux termes avant d'entrer plus spécifiquement dans l'étude de cas proposée. Du point de vue de l'espace dédié au musée, l'espace muséologique englobe tous les espaces et toutes les fonctions rattachées au musée à leur état brut. Un musée est un rassemblement de fonctions, il englobe entre autres les dépôts, les salles d'administrations, les salles de recherche et de préservation, les espaces d'expositions, le magasin, et toutes les autres activités. Ainsi, l'espace muséologique - peut être défini par l'espace en tant que tel qui hébergerait toutes ces fonctions. Par ailleurs, l'espace - muséographique -, qui a été habité par des histoires expographiques, des scénographies spécifiques et des activités spatiales, devient le médiateur qui rapproche le personnel muséal et les visiteurs de la collection et du musée. Ce travail interprété par le muséographe « apprivoise » alors l'espace et le rend familier et adapté à ses utilisateurs. II se différencie donc des autres lieux muséaux et met en valeur l'identité et l'histoire. Pour concrétiser encore plus cette comparaison, nous nous referons aux deux mots anglais « house » et « home ». " House », qui définit une maison dans le sens brut du terme, et «Home », qui revoie à la maison de l'individu, où maints évènements ont eu lieu, un espace où émergent des souvenirs, un espace où l'individu s'identifie, un espace qu'il s'approprie.

Dans cet article nous nous intéresserons à l'espace muséal et son importance sensorielle et communicationnelle au sein des musées universitaires. Il s'agira d'étudier les configurations architecturales ainsi que les techniques de présentation et de traitement de l'espace muséal afin de transformer cet espace muséologique en un espace muséographique, de mettre en place une meilleure communication entre les objets et le visiteur et d'intégrer le musée dans la vie estudiantine - voire, selon notre analyse, ce qui déterminerait la beauté et l'efficacité d'un musée ou d'un espace muséal. Quels sont les critères qui déterminent si une salle d'exposition ou un musée sont réussis ? Comment transformer l'espace muséologique en espace muséographique approprié par ses utilisateurs?

\section{Les musées universitaires}

Depuis le début du XXle siècle, les musées universitaires connaissent un grand succès, souligné par la création d'un Comité International pour les musées et les collections universitaires (UMAC) au sein du Comité International des Musées (ICOM) 
(SOUBIRAN et alii 2009, p. 6). Ce comité affirme qu'il importe grandement de reconnaître le travail qu'il reste à accomplir par les professionnels des musées universitaires et de soutenir la revitalisation des structures muséales dans le monde académique.

Le musée universitaire cache des trésors à redécouvrir. II est temps de recomposer un musée qui regroupe de riches collections, afin que l'université joue son rôle de transmetteur de culture en protégeant, en exposant et en étudiant cette richesse. II est aussi temps de concevoir un modèle de "Musée d'enseignement ». Nous pensons notamment au Musée des Arts et Métiers du Conservatoire National des Arts et Métiers (CNAM) à Paris, en France. Le Musée des Arts et Métiers du CNAM est un établissement public, sous la direction du Ministère de l'Enseignement Supérieur et de la Recherche. Ce musée, par son caractère professionnel, culturel et scientifique, abrite une précieuse collection sur l'évolution des savoirs techniques, la communication, la construction, les matériaux, les instruments scientifiques, l'énergie, ainsi que les transports et les mécaniques (CNAM s. d.).

Rénové en l'an 2000 pour donner accès au large public à des formations culturelles et professionnelles, le Musée des Arts et Métiers est ouvert aux étudiants et chercheurs pour approfondir la connaissance des métiers et des arts anciens. II utilise une pédagogie par l'objet qui permet aux visiteurs, ouvriers professionnels et autres, de redécouvrir les anciens objets et de réutiliser les anciennes machines afin de concevoir de nouveaux objets. II utilise, au lieu de la parole, la science des faits, et permet aux anciennes machines de reprendre leur mouvement et de fonctionner à nouveau sous les yeux des visiteurs. Les collections universitaires sont généralement connues pour leur grande diversité : diversité d'objet, de collections, de sources, de thèmes, etc., qui constitue une richesse à la fois attirante pour les recherches scientifiques, mais aussi pesante pour ce qui en est de la gestion, l'entretien et la coordination. Cette diversité pose aussi le problème de l'espace, des lieux d'exposition et de rangement. L'enjeu de l'espace architectural, plus précisément dans les musées universitaires, s'inscrit dans la question de la complexité du public et de la présence de ces établissements, généralement, au sein d'un campus universitaire. Pour y répondre, il est nécessaire d'œuvrer à trouver les fonds nécessaires à la préservation et la mise en valeur des collections, mais aussi de monter des projets culturels et d'effectuer des recherches scientifiques.

Dans le but d'approfondir le rôle de l'espace et de l'expographie dans l'amélioration de la vie muséale et étant donné que les différents cas de musées universitaires sont nombreux et variés, nous étudierons, plus spécifiquement, le cas du Musée Archéologique de I'Université Saint-Esprit de Kaslik, Liban (USEK).

Le Musée de I'USEK, Musée Archéologique Universitaire (MAU), possède une collection unique comprenant entre autres éléments un outillage lithique de différentes techniques 
qui relate l'évolution préhistorique du Paléolithique et Épipaléolithique (1,5 million BP-12 000 BP) jusqu'à la sédentarisation au Néolithique (6900 av. J.-C, 4500 av. J.-C.) au Liban², ainsi qu'une grande collection de verrerie romaine, céramiques, monnaies, objets cultuels des anciennes civilisations jusqu'au christianisme (spécifiquement les maronites), parures et autres. La collection du MAU provient essentiellement du Liban. Elle retrace I'histoire du pays depuis la Préhistoire, jusqu'à la période ottomane et compte près de 4000 objets archéologiques. Récemment, suite à une intention de donation d'une collection de verreries qui comprend plus de mille objets, le MAU se trouve devant la nécessite de réaménager son espace muséal. Ce projet aura pour but de remettre en valeur la collection toute entière, en particulier celle des verreries, et de réintégrer l'expérience muséale au sein de la vie universitaire, avec une ouverture au grand public.

\section{Problèmes et demandes actuels du Musée archéologique de I'USEK}

\subsection{Analyse de l'espace existant}

Maints problèmes d'intégration et de conservation trouvent leur solution dans une bonne expographie et dans l'aménagement ordonné de l'espace. Le musée universitaire, et spécialement le MAU, se trouve lié physiquement et administrativement à un établissement universitaire, ce qui présente des points forts et en même temps compliqués. Toutefois, du point de vue volumétrie et architecture, il y a deux obstacles au réaménagement et à la mise en valeur du MAU : la petite superficie du musée et le lieu de son emplacement à l'intérieur de l'université. Le MAU se trouve au sous-sol de la Faculté des Beaux-Arts. De l'extérieur, il passe inaperçu, caché par le volume du restaurant universitaire. Pour y arriver il faut y accéder par un grand escalier, donnant sur un patio, qui mène à un amphithéâtre, à la cafétéria et au musée. Son entrée principale, qui est con située d'une simple porte en bois, ne donne aucun indice sur ce qui se trouve à l'intérieur. De l'extérieur, l'indication de la présence d'un musée archéologique n'est ni claire, ni bien visible, tant pour les visiteurs que pour les étudiants. D'autre part, la superficie intérieure se réduit à une grande salle de 40 mètres de long et 18 mètres de large, reposant sur douze colonnes centrales et, à son extrémité gauche, se trouve un petit dépôt. L'espace n'était pas conçu pour abriter un musée, au début la salle d'exposition actuelle était une grande salle réservée à l'atelier d'architecture pour le travail des élèves. De même, dans cet espace nous ne trouvons aucune ouverture qui permette la pénétration de la lumière naturelle. Le plafond, les colonnes et les murs sont teints en blanc et le sol est couvert de carreaux gris bleu. Les vitrines présentes sont blanches et principalement de deux types: des vitrines tables et des vitrines murs (fig. 1).

\footnotetext{
2 En 2004, Georgette Habisse, veuve de feu Paul-Émile Guigues (1868-1930), lègue au MAU la collection de son
} mari. 


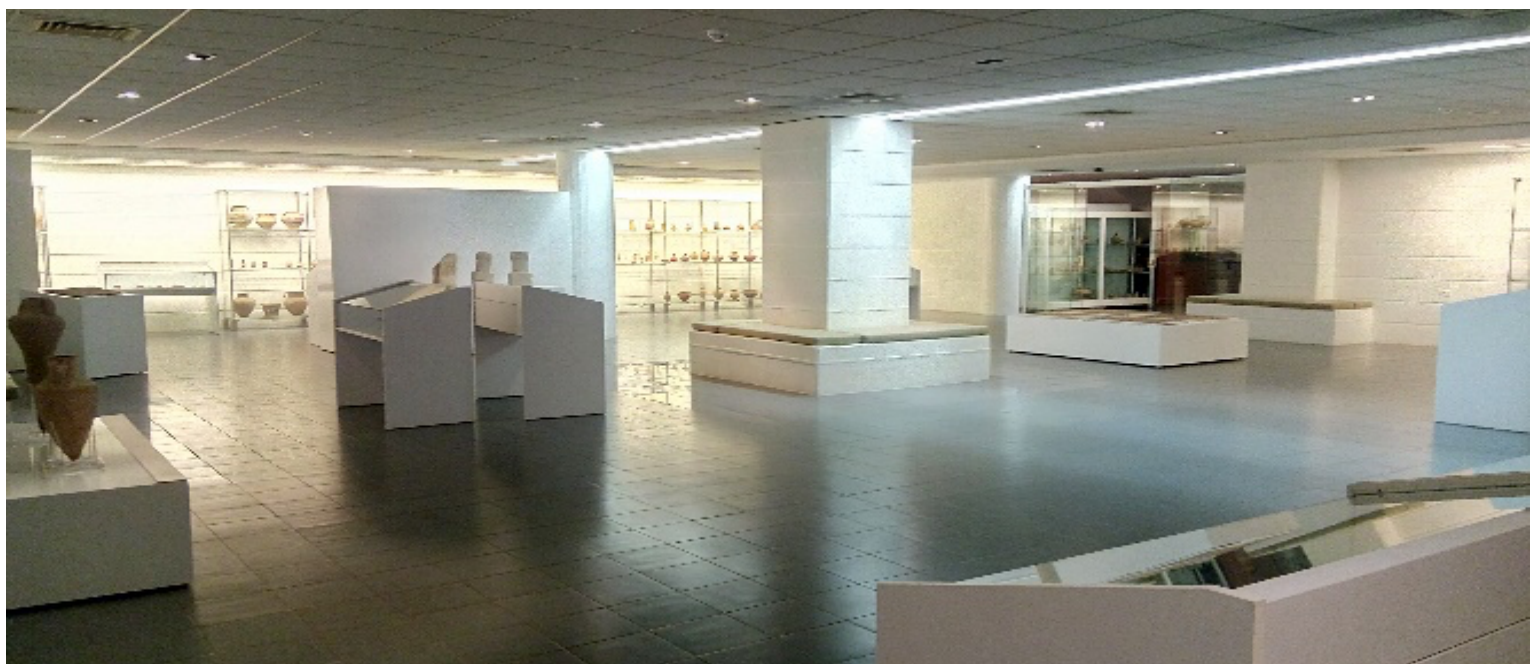

Figure 1 - Espace intérieur du MAU, 2017. Photo : Gilbert Nicolas.

Dans ce genre d'espace, les points positifs sont les suivants : la liberté dans le choix du parcours, la distribution équilibrée des sous-espaces d'exposition et le manque de contraintes devant la distribution des vitrines et la division des espaces. En revanche, les points négatifs sont le manque de caractère architectural et l'absence d'ouvertures et de fenêtres qui laissent pénétrer la lumière et l'aération naturelles.

Le musée ne constitue pas alors un bâtiment séparé que nous pouvons bien distinguer du reste des facultés. De plus, la présence des autres bâtiments, du seul restaurant du campus et de l'amphithéâtre étouffe - pour ainsi dire - l'entrée du musée (fig. 2). Par contre ces deux éléments peuvent être un point fort pour promouvoir le musée et le rendre plus fréquenté. Nous nous trouvons ici devant plusieurs contraintes architecturales d'où notre étude viendra répondre tout en respectant la collection, l'expographie muséale et la communication avec l'entourage universitaire. Notre proposition favorisera les relations entre, d'une part, les étudiants et le corps muséal et, d'autre part, entre les étudiants et les objets exposés. Nous essayerons aussi, dans la mesure du possible, d'activer et d'attirer des visiteurs de l'extérieur de l'Université. Cette approche a comme finalité l'intégration du MAU dans les enjeux sociaux universitaires contemporains par la diffusion des connaissances au sein de la population étudiante, qui constitue les principaux acteurs de demain, mais aussi au sein du plus large public. 

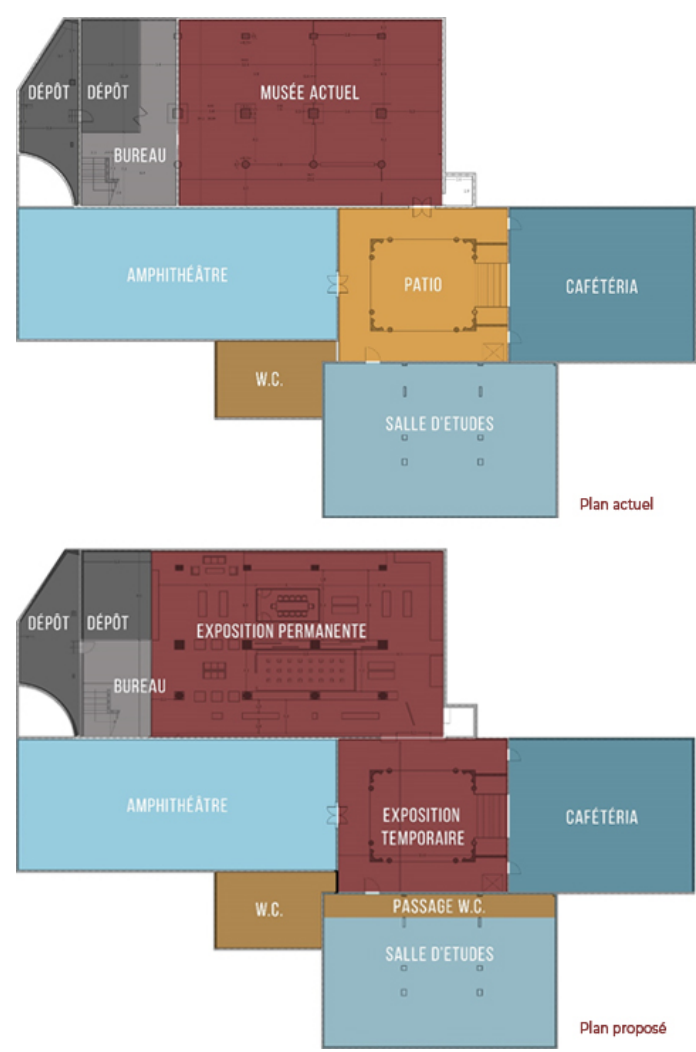

Figure 2 - Plan actuel du MAU, son entourage et le plan proposé pour l'aménagement du MAU. Photo : Gilbert Nicolas.

\subsection{Analyse au sein des élèves de l'université}

Dans le but d'établir une réintégration et une revitalisation du MAU, nous avons effectué une enquête auprès de 250 étudiants de I'USEK pour en déterminer les principaux problèmes et essayer par le biais de la muséographie de trouver des solutions et des propositions adéquates (tableau 1).

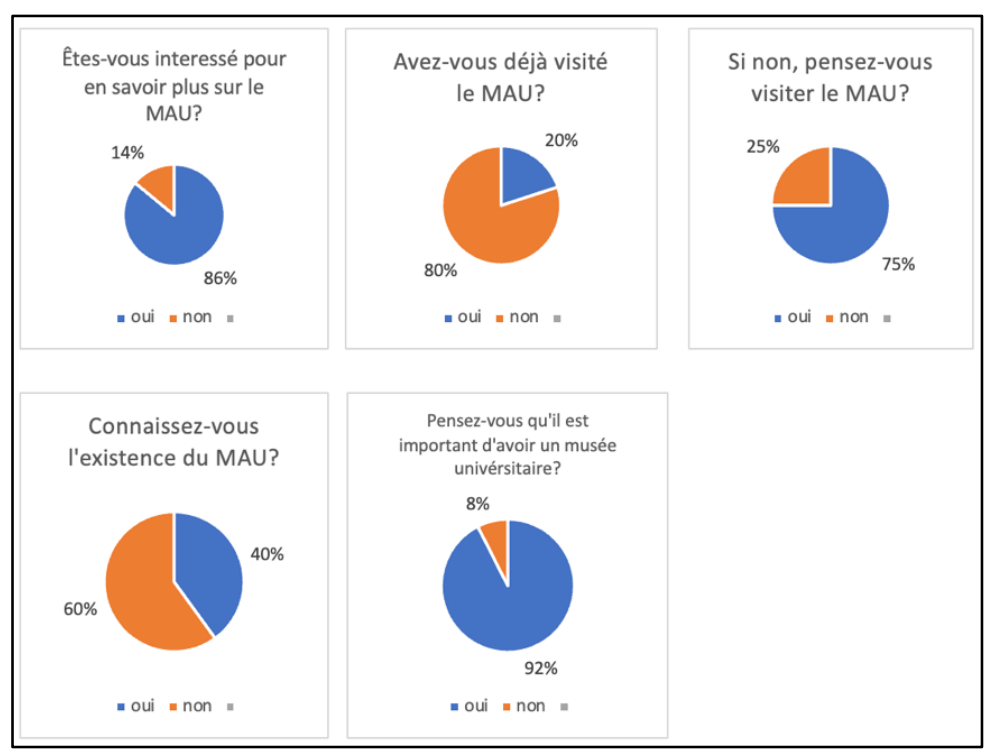

Tableau 1 - Résultats de l'enquête statistique menée auprès de 250 étudiants de I'USEK. Photo : Gilbert Nicolas. 
Les chiffres issus de notre enquête statistique nous montrent que la majorité des étudiants (60\%) ne connaissent pas le MAU. Par contre, 92\% des élèves pensent que c'est important d'avoir un musée universitaire. De même, un grand nombre d'élèves sont au courant que leur université abrite un musée archéologique, mais ne l'ont jamais visité. Presque le même nombre d'élèves se trouvent intéressé par la visite du MAU et par l'envie d'en savoir plus.

Sur ce questionnaire, nous avons ajouté des questions pour demander aux élèves dans quelles mesures la présence du musée les aide-t-elle dans leur cursus éducatif ? Et quelles sont leurs propositions pour l'intégration du musée dans la vie universitaire ? Les étudiants insistent sur l'importance d'avoir un musée universitaire et sur le fait que ce soit un musée qui préserve et conserve des objets archéologiques d'une grande valeur culturelle et historique, au sujet desquels ils ont la curiosité d'en savoir plus. Les propositions des étudiants de I'USEK pour améliorer leur musée universitaire et l'expérience muséale se résument comme suit: d'abord, la plupart des étudiants ont pointé du doigt le fait que le musée ne doit pas être enfoui dans le sous-sol d'une faculté. Ils pensent que le musée n'est pas visible et qu'il se trouve loin de l'activité quotidienne de leur vie universitaire. Les étudiants demandent une rénovation du musée et de son espace expographique et une intégration concrète de la Faculté d'architecture et d'histoire de l'art par des projets qui concernent le MAU. Ils pensent aussi que la création d'événements de design et d'expositions contemporaines aideraient à les attirer au musée et à le faire visiter. De plus, ils proposent d'organiser des visites durant le semestre qui seraient inclues dans leurs études, afin de découvrir le musée et sa richesse. Ils ont insisté sur l'intégration de la recherche muséale et expographique dans le cursus universitaire.

Quant à la question de l'importance du musée dans les recherches universitaires, la plupart des étudiants ont affirmé ce qui suit : ils ont proposé des expositions sur des thèmes et des sujets contemporains qui peuvent aider dans les nouvelles recherches historiques et scientifiques. De même, ils ont demandé d'avoir un musée qui soit une plateforme de discussion et d'études. Les élèves assurent également que le musée peut être une source d'inspiration pour la créativité et pour la découverte. Il joue un rôle moteur pour la connaissance, l'enseignement, la motivation et l'interaction.

Dans son état actuel, le musée universitaire de I'USEK répond partiellement aux demandes des étudiants et s'engage timidement dans la vie universitaire. Malgré quelques séminaires et évènements autour de la muséologie comme celui qui a été organisé en mars 2013 en collaboration avec le Pôle Méditerranée de l'Université Paris 8 Vincennes - Saint Denis et I'Université Paris Ouest Nanterre La défense (BULLETIN DE L'USEK, s. d.) sur le thème « Le musée qui devient catalyseur de recherche et d'enseignement en muséologie », beaucoup de travail reste à accomplir. Ce Musée se trouve, devant la nécessité de revivifier son espace 
muséal, de préparer une étude globale pour son expographie et de réengager ses collections au sein de la vie des étudiants.

Le projet se résume à l'intégration du musée dans la vie universitaire. Cela se traduit par l'intégration des collections existantes dans un nouveau parcours muséal attirant et animé, en premier lieu pour les étudiants, mais aussi pour les visiteurs et les chercheurs. Nous pourrons commencer cette réintégration par l'étude de l'espace architectural, extérieur et intérieur, qui s'engage dans le processus communicationnel de l'exposition et participe à donner du sens à la cohabitation des objets archéologiques présents malgré leur diversité thématique. Ensuite, nous proposerons un plan expographique (conception du trajet, promenade, signalétique, aménagement de l'espace, etc.) et communicationnel qui rapproche la vie universitaire du musée et des objets archéologiques tenant compte de la richesse des domaines sur le campus. II s'agit donc d'étudier les configurations spatiales ainsi que les techniques de présentation et de traitement de l'espace qui permettent la mise en place de la communication et la production du sens dans l'exposition.

\section{Transformation de l'espace muséographique en espace muséologique}

\subsection{Les critères pour une conception expographique réussie}

Commençons par quelques points qui montrent l'importance d'une bonne conception expographique dès l'élaboration d'un projet muséal, afin de transformer un espace muséologique en un espace muséographique captivant. Pour pouvoir aboutir à un musée attirant et agréable à visiter, de même que pour avoir un espace muséographique hautement fréquenté et intégré dans la vie quotidienne de l'université et de la région, il nous semble important, après avoir travaillé la nouvelle scénographie muséale, d'intégrer des espaces de lecture, d'études et de recherches. Nous choisissons de souligner l'importance que donne l'effet architectural et spatial sur la vie muséale. Pour cela, nous présenterons six critères globaux qui sont essentiels pour la conception d'une exposition réussie (NICOLAS 2020, p. 48-49):

1- L'ordre. L'ordre signifie équilibre visuel et physique, une disposition ordonnée par une sorte de répétition, une symétrie ou même une composition visuelle rythmée et équilibrée qui aide le visiteur à se sentir dans un espace sécurisé, familier et reposant. Au contraire, un espace désordonné donne une impression d'insécurité et de manque de stabilité. En contrepartie, trop de symétrie et d'ordre donnent le sentiment de se trouver dans un musée excessivement systématique, cartésien, vide de toute émotion et de toute âme, il reflète donc beaucoup de rigidité ce qui influe négativement sur la visite. L'idéal consiste à trouver le juste milieu entre ordre et variété, en choisissant une composition expographique ordonnée et un jeu scénographique rythmé et varié. Dans le projet proposé pour la conceptualisation 
du MAU, l'ordre est assuré par le choix des couleurs de l'espace architectural, les luminaires et les matériaux utilisés tout au long de la grande salle d'exposition. La scénographie des sous-espaces subit un changement raisonnable selon les thèmes exposés, ce qui introduit un changement ordonné dans le parcours muséal. Ce mélange entre ordre et diversité influe positivement sur la qualité de la visite et rompt la monotonie dans la visite.

2- La vie à l'intérieur des musées. Souvent, les visiteurs des musées universitaires apprécient se retrouver avec d'autres personnes pour discuter de l'art, de la science et de l'histoire et échanger leurs idées. Le rôle de l'espace muséal dans ce cas se manifeste par l'encouragement des rencontres sociales entre les visiteurs, il se transforme en un lieu d'échanges et de discussions. La présence des meubles dans les salles d'exposition sera étudiée sous cette dimension. De plus, nous pouvons transmettre aussi cette vie conversationnelle à l'extérieur du musée, que le public puisse voir de l'extérieur ce qui se déroule à l'intérieur. Nous avons travaillé ce point par la médiation que l'espace architectural et l'expographie peuvent assurer. Dans cette perspective, nous avons créé des liens entre la vie muséale et la vie estudiantine afin d'adapter le musée aux enjeux contemporains de l'université et du pays. Nous avons proposé une salle d'étude et de lecture à l'intérieur du parcours muséal pour inciter les étudiants à s'approprier l'espace et à l'utiliser plus fréquemment. La création de cette salle à l'intérieur de la salle d'exposition garantit une relation sociale entre les étudiants et les visiteurs et une médiation culturelle visuelle et spatiale entre ces derniers et les objets exposés. Notons également que la création des coins salons, où le visiteur peut s'asseoir, contempler ou discuter, favorise les échanges scientifiques et culturels. De la même manière, la relation entre les conservateurs et les chercheurs du musée et le public est assurée dans le parcours où le visiteur peut voir, à travers les vitres, le corps muséal lors de son travail quotidien. Il peut aussi voir les réserves, les dépôts et les salles de réunions du personnel du musée, ainsi ces derniers sont eux aussi exposés dans la promenade muséale et transmettent les connaissances et les coulisses du travail muséal aux publics. Enfin, par la création d'un Hall extérieur pour les expositions temporaires, nous avons produit un espace de rencontre devant le musée. La salle d'exposition temporaire déplacée en dehors des murs du musée, dans le hall extérieur qui sert l'amphithéâtre, la cafétéria et les salles d'études, est un signe important que le musée s'ouvre aux étudiants pour les inviter à entrer et rompre la barrière physique qui les sépare.

3- L'échelle de l'espace. Dans un musée, ce que cherche l'architecte muséographe se traduit par un équilibre entre, d'une part, un grand espace qui abrite un nombre important de visiteurs et d'œuvres exposés, et, d'autre part, un espace intime, à 
échelle humaine. L'homme cherche à maîtriser l'espace qui l'entoure, qu'il soit fait par lui ou bien pour lui. En pénétrant dans cet espace, l'homme commence à l'évaluer, à marcher en cherchant les connexions, les successions, la continuité, ou la discontinuité. Selon J. Cousin, un espace vaste et majestueux renvoie à un sentiment de petitesse et de perte qui introduira le visiteur dans un état d'insécurité et de confusion (COUSIN, 1980, p. 143-136). En contrepartie, un espace très étroit suscite des sentiments d'oppression et d'encombrement. Dans un espace équilibré dans son organisation spatiale le visiteur aura alors le sentiment d'être bien entouré, sécurisé et, simultanément, il aura l'esprit aéré et détendu. Le MAU est à l'origine une salle de dimensions moyennes, sa transformation en musée universitaire a obligé l'espace restreint à s'adapter à la collection muséale et vice versa. L'échelle architecturale est donc relativement petite par rapport à la grande collection archéologique du musée. Nous remodèlerons tout l'espace en créant des liens entre l'extérieur et l'intérieur du musée. Le musée sortira du cloisonnement de ces quatre murs pour rejoindre les étudiants à l'extérieur dans le hall central que nous avons déjà mentionné dans le point précèdent. De même, à l'intérieur, nous avons créé des espaces thématiques qui favorisent les interrelations entre les différentes époques et collections afin de renforcer l'esprit de recherche historique et chronologique. Notre projet, par le choix des couleurs sombre, de la lumière directe et indirecte et de la division de l'espace en petits espaces semi-ouverts, donne l'impression que la salle est plus large que la réalité. Cela aide le visiteur à dépasser le sentiment d'encombrement, à se sentir bien entouré physiquement et en sécurité.

4- L'orientation du visiteur et son esprit de curiosité. Nous trouvons plusieurs genres d'espaces: par exemple, des espaces limités ou vastes, concaves ou convexes, ouverts ou fermés, hauts ou bas, transparents ou opaques. Toutes ces caractéristiques, et bien d'autres, rendent l'expérience muséale intéressante du fait qu'elles soient combinées, tout en respectant une continuité et une certaine harmonie entre eux. Une grande superficie d'exposition contenant de petits espaces dissimulés, des espaces périphériques, des chambres thématiques annexes, de petits lieux de repos, d'échange, ou de recherche, poussent le visiteur à la découverte et suscite sa curiosité. Dans notre projet de réorganisation du MAU, l'orientation est assurée par le trajet qui est globalement guidé. En même temps, le choix de laisser la liberté au visiteur de tracer sa visite dans les sous-espaces d'exposition apaise le flux dicté dans le musée et favorise les échanges sociaux. De plus, à la fin du parcours, nous avons changé la couleur des vitrines en couleur bordeaux, qui diffère totalement de la scénographie antérieure, pour modifier l'esprit du visiteur et le pousser à en découvrir davantage. Le visiteur semble être poussé à la découverte à cause du changement opéré dans la scénographie et la couleur. 
5- L'organisation des objets exposés. L'organisation est une série décroissante ou croissante d'éléments classés selon leur grandeur ou valeur (Larousse pratique 2003, p. 722). De même, l'organisation d'objets dans un musée peut s'établir selon différentes formes et critères selon l'identité du musée et sa mission propre. Nous trouvons normalement un classement hiérarchique, thématique ou parfois chronologique mais on trouve aussi d'autres formes de division d'espaces comme par couleur, forme ou matériaux. La logique de classement est choisie en fonction du récit muséographique. Le récit muséographique traverse la promenade du projet en indiquant lui aussi, par le biais de l'histoire racontée, la disposition des pièces de l'exposition. M. Bal identifie le récit de l'exposition et l'itinéraire comme les coordonnées qui aideront l'objet muet à communiquer avec le visiteur et à prendre, dans l'espace, tout son sens, à la fois large et précis (BAL, 1996). Les espaces sont à la fois physiques et symboliques: ce sont des espaces mutuels, occupés par des objets et des visiteurs, des textes et des installations. Il existe aussi, à travers la technologie, des espaces virtuels par l'intermédiaire des écrans et de la réalité virtuelle qui dépasse l'espace physique du musée. Dans notre projet du musée archéologique, le mode de classement choisi est thématique et respecte la hiérarchie des objets. Cela aide le visiteur à réaliser des concordances claires entre les objets, leurs fonctions et l'époque de leur utilisation. De même, ce classement répond directement à l'identité archéologique et universitaire du musée et facilite la recherche et la transmission des informations aux chercheurs, aux élèves et aux visiteurs. De plus, notre structure architectural présente une séparation physique entre les différents espaces du musée, notamment entre les espaces consacrés au travail administratif, à l'exposition permanente et à l'exposition temporaire. L'histoire expographique, que nous proposerons dans la partie suivante, aidera considérablement à l'organisation de l'espace.

6- L'identité du musée. L'identité comporte un sens large, puisqu'elle renvoie à l'identité socioculturelle, fondamentale pour déterminer la place du musée dans sa communauté. Étant donné sa mission culturelle et éducative, un musée rassemble une communauté de citoyens dans le partage d'une culture commune (CHAUMIER 2003, p. 22). La collection même du musée peut refléter l'identité profonde d'un peuple, son histoire, son système d'échanges économiques et politiques, elle soude donc les liens d'appartenance à la communauté. Le musée, par son architecture, doit avoir un caractère fort, précis et bien identifiable qui le différencie des autres bâtiments culturels et des autres musées, même s'il rejoint dans son identité profonde tous les autres musées. Le MAU est un musée archéologique et universitaire, son identité éducative et conservatrice est claire tout au long de notre 
projet architectural par la coexistence des salles de travail et d'étude et du trajet muséal et de l'exposition.

L'identité et l'histoire de l'université peuvent être façonnées par leurs musées et leurs collections, qui jouent un rôle important dans l'évolution de l'enseignement supérieur. Grâce au musée universitaire, la recherche scientifique, I'histoire, l'archéologie et leur rapport avec les thèmes contemporains seront un centre d'intérêt du grand public. De cette manière, une contribution à l'éducation collective de la société sera atteinte. Les plateformes muséales forment un espace concret pour l'évolution des études et leur diffusion, et l'expographie vient aider physiquement la réalisation de cette mission.

\subsection{Scénographie et parcours muséal}

Dans notre cas, le musée est divisé en plusieurs parties, il raconte plusieurs histoires individuelles mais qui, dans leur ensemble, entretiennent un lien de continuité. Notre histoire décrit une démarche archéologique qui porte le visiteur dans plusieurs lieux et à travers plusieurs états d'âme. Nous proposons au visiteur de commencer sa promenade par le hall extérieur, baigné par la lumière naturelle, comme s'il commençait à pénétrer dans I'histoire par un seuil qui sépare l'extérieur de l'intérieur, un extérieur lumineux qui mélange plein de couleurs vers un endroit inconnu, sombre et mystérieux. Une invitation à voyager dans le temps et à entrer dans une aventure archéologique muséale.

L'élément central, au début de l'itinéraire formé par une bande circulatoire qui semble émerger du sol, donne l'impression que le visiteur traverse une fouille archéologique ou des ruines sortant du sol, et des objets commencent alors à apparaître devant lui (fig. 3).

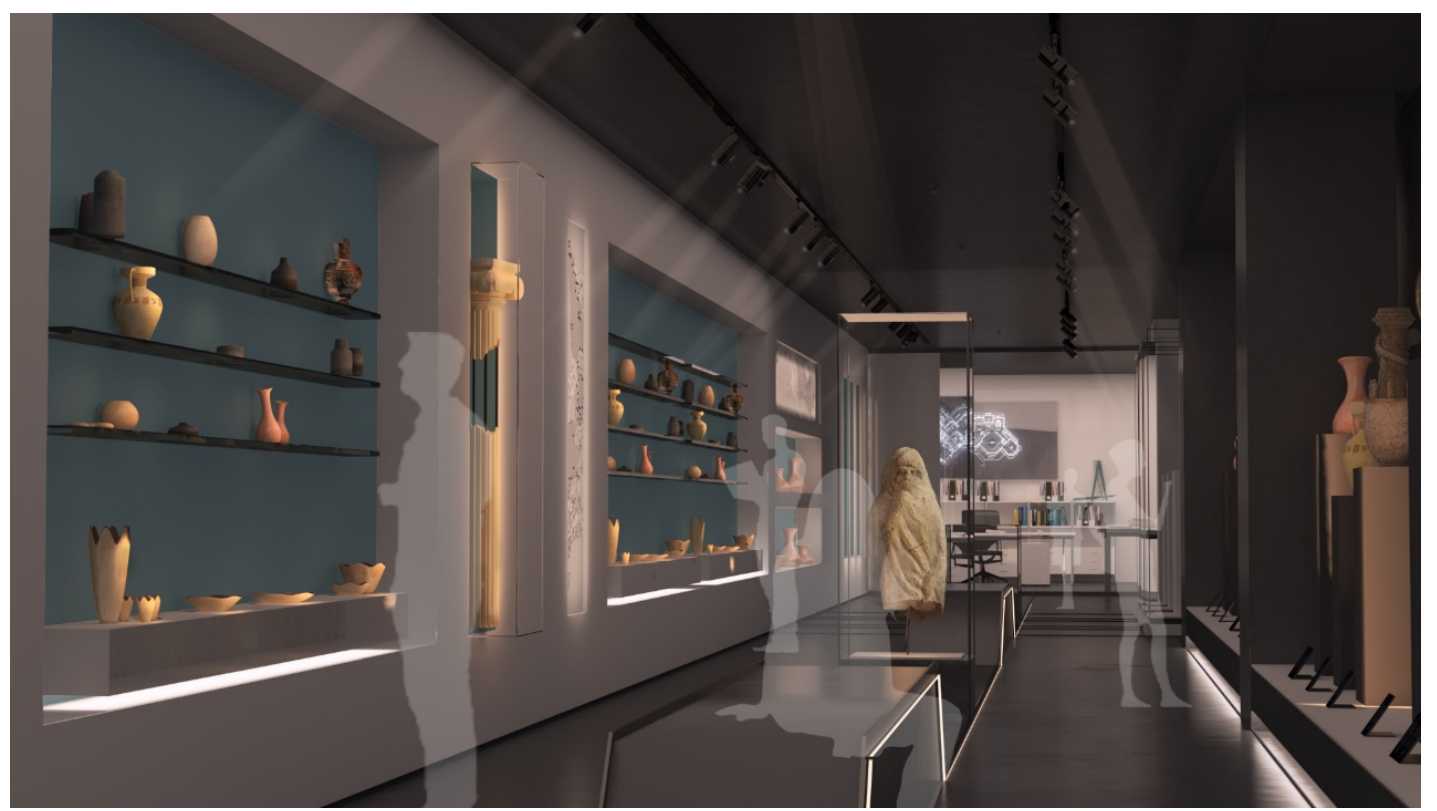

Figure 3 - Représentation 3D de la première salle d'exposition du MAU. Photo : Gilbert Nicolas. 
Le visiteur pourra observer l'évolution de ces objets au cours des époques et le changement des formes et des dessins selon chaque civilisation. À la fin de cet espace, nous avons placé le bureau du conservateur du musée avec un atelier de travail pour permettre aux visiteurs de regarder le travail de préservation et de consolidation des objets. Durant son parcours, le visiteur aura la chance de voir, à travers un mur vitré, le grand travail à l'œuvre derrière cette exposition.

L'histoire se poursuit en rentrant dans la «salle du trésor », la «Black Box», qui contiendra la collection de verreries. Lors de cette expérience, le visiteur pénètre dans un lieu mystérieux en contemplant des objets de grandes valeurs, et il avance pour entrer dans un lieu sombre et inconnu. Désormais, il ne se trouve plus dans un lieu où il découvre des objets archéologiques, mais dans un cheminement vers une sorte de " sœur » des temples des anciennes civilisations. Nous voulons ainsi donner au visiteur l'impression qu'il voyage dans le temps. Une fois à l'intérieur de la «Black Box», le récit change totalement pour transporter le visiteur d'un espace blanc, accueillant et vaste, en un endroit sombre, noir et secret. À l'intérieur de cet espace, seuls les objets de verrerie seront illuminés et tout l'espace sera plongé dans l'obscurité (fig. 4).

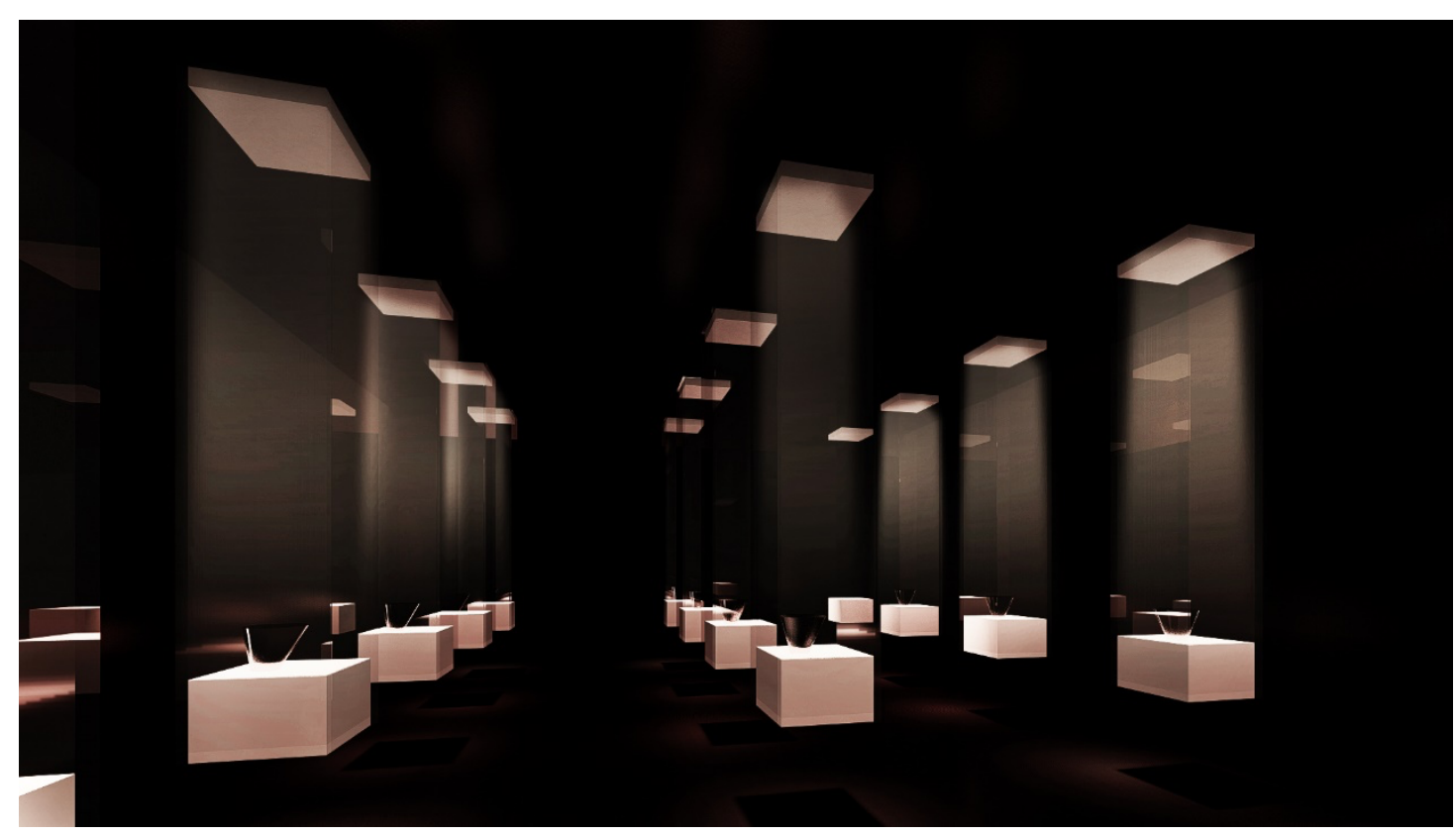

Figure 4 - Intérieur de la «Black Box». Photo : Gilbert Nicolas.

Cet effet s'inscrit dans cette étape du parcours pour donner l'impression que nous sommes en train de pénétrer dans les profondeurs de l'histoire archéologique et d'entrer dans cette dimension inconnue des fouilles où, durant sa recherche, l'archéologue se trouve plusieurs fois face à une sorte de mystère. Nous transmettons au visiteur ce sentiment d'« inexploré » lorsqu'il ne s'attend pas à ce que, pendant une nouvelle fouille, il découvre quelque chose de nouveau. Choisir de mettre uniquement la lumière sur les objets exposés pourrait être comparé aux petites découvertes faites tout au long d'une fouille archéologique. Une fois 
ramassés et assemblés, ces objets permettront à l'archéologue de former sa synthèse et de mettre en lumière une nouvelle découverte qui était enfouie dans l'obscurité durant des millénaires : c'est là l'effet désiré par la scénographie de cette étape. À la troisième étape, nous proposerons aussi au visiteur de jeter un coup d'œil, à travers un mur vitré, aux dépôts du musée, et également aux bureaux et ateliers. Nous avons intégré cette étape pour montrer que la collection du musée ne se limite pas seulement aux objets exposés dans les vitrines, mais qu'elle s'étend au-delà des salles d'expositions. Le visiteur entre ensuite dans un espace d'exposition dont la pièce centrale est une salle de réunion vitrée (fig. 5).

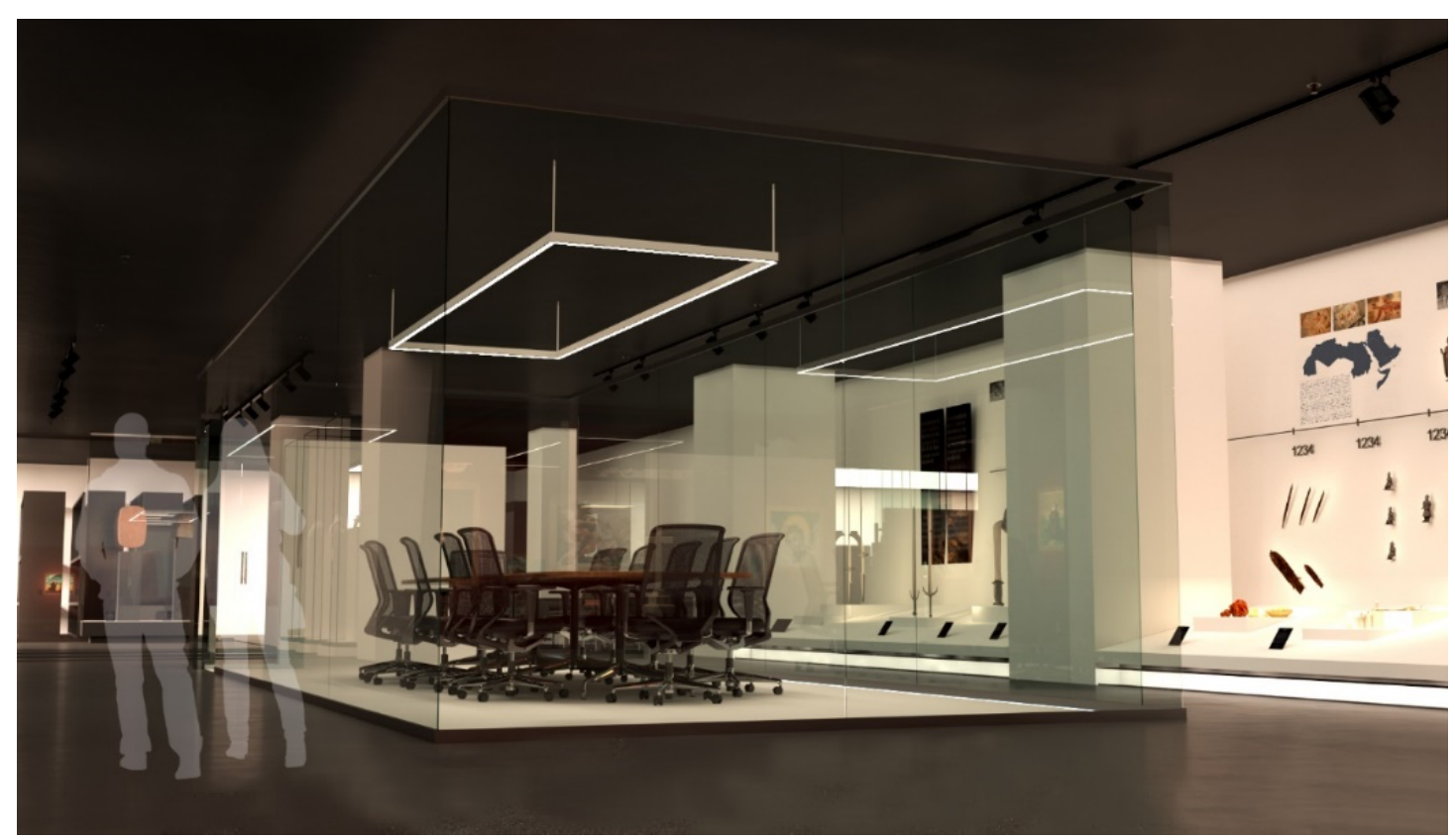

Figure 5 - Représentation tridimensionnelle de la salle de réunion et des murs de projection. Photo : Gilbert Nicolas.

Cette étape est le lieu de la recherche dans l'étude archéologique, et une grande vitrine murale est visible de l'intérieur de cette salle de réunion. Arrivant à la dernière étape du parcours proposé, le visiteur se trouve devant une large vitrine de couleur bordeaux qui diffère entièrement de la totalité des autres espaces. Lors de cette étape, nous rencontrons la collection cultuelle et funéraire avec la collection des tableaux religieux que possède le musée. Dans cet endroit, la vitrine se transforme en une chambre qui permet au visiteur de pénétrer progressivement à l'intérieur d'un nouvel espace par une marche qui insiste sur cet effet ascendant. Nous avons créé la vitrine comme une sorte de grande niche pour procurer un sentiment d'enfermement afin de contempler et de prendre le temps d'admirer les objets exposés (fig. 6). 


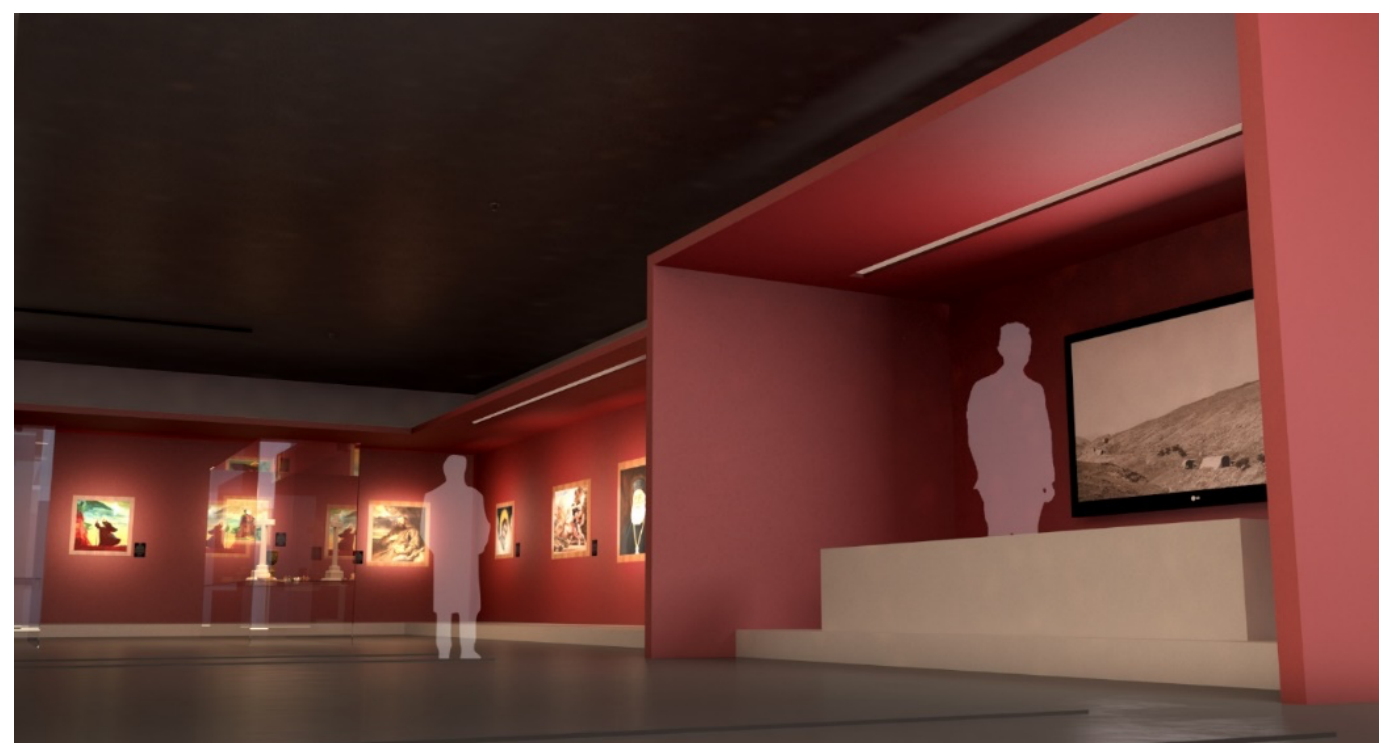

Figure 6 - Représentation 3D de la vitrine niche. Photo : Gilbert Nicolas.

Le sol du musée sera teint en noir, tout comme le plafond, afin de focaliser le champ de vision des visiteurs uniquement sur les vitrines blanches et les objets exposés. Ce mélange de noir et de blanc, avec les couleurs choisies comme fond pour quelques vitrines, renforcera le sentiment d'un espace vaste et mettra les objets de la collection en valeur.

Cette division de l'espace et de la circulation, entre liberté et contrainte, aide les visiteurs à rester captivés durant leur visite et à toujours vouloir avancer dans l'espace pour en découvrir plus; elle les incite aussi à venir revivre cette expérience. Voici ci-dessous l'axonométrie qui représente l'organigramme pour avoir une meilleure idée de la relation des différentes fonctions qui constituent le MAU (fig. 7).

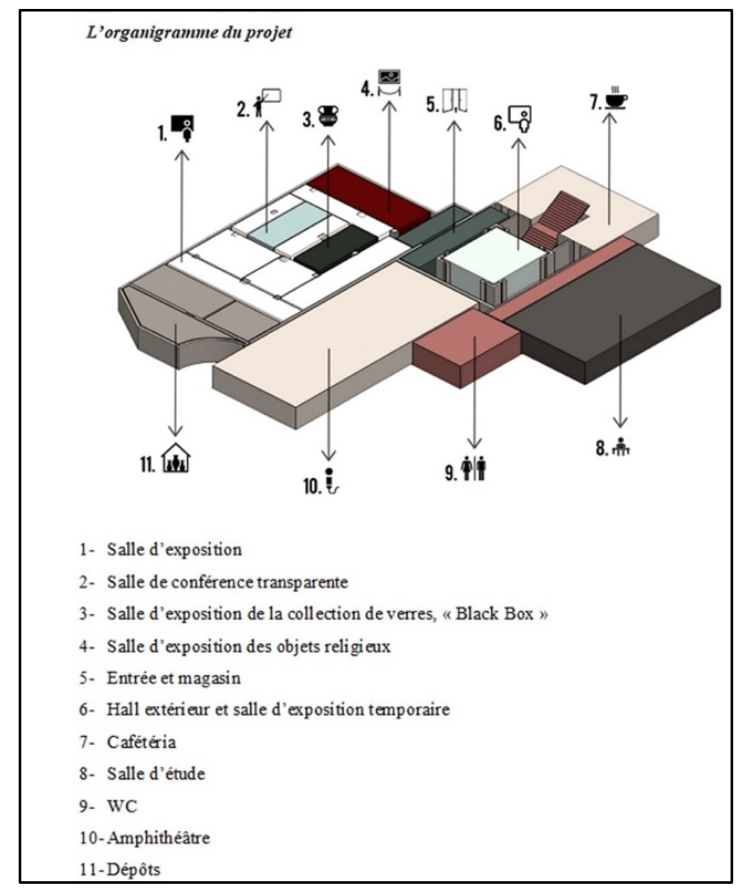

Figure 7 - Organigramme du projet de conceptualisation du MAU. Photo : Gilbert Nicolas. 


\section{Conclusion}

Le travail expographique que nous venons de proposer pour le MAU résulte de l'importance prise par les différents éléments qui constituent les espaces extérieurs et intérieurs des musées. Par cette conceptualisation du MAU, il répond concrètement à la question de la transformation de l'espace muséologique en un espace muséographique. L'espace sera adopté par les visiteurs et fera partie intégrante de leur vie quotidienne. II sera un lieu privilégié et revisité plusieurs fois comme un refuge culturel et scientifique. L'étude de différents musées et expositions montre la grande importance de l'interrelation entre espace architectural, exposition et public.

L'importance du rôle des musées universitaires dans la vie étudiante et leur travail face aux enjeux contemporains de la société entre en résonnance avec cette revitalisation du MAU. Ce renouvellement sera-t-il utilisé pour remodeler l'image de l'université et l'ouvrir à de nouveaux modèles d'éducation? Le musée universitaire peut-il être un outil de renouvellement des partenariats universitaires publics et privés? Peut-il être un moyen qui distingue par sa valeur l'université et attire les chercheurs locaux et internationaux afin qu'ils réalisent des études approfondies sur l'histoire du pays et sur son avenir?

«(...) l'architecture est le musée : nettement, la configuration architecturale donne le sens au musée. L'architecture définit les règlements et les directions de vue de l'homme, conceptuellement et physiquement. Elle ne concerne pas uniquement les expositions mais sculpte aussi l'expérience de la visite » (GIEBELHAUSEN 2006, p. 42). 


\section{Liste des abréviations}

UMAC : les Musées et les Collections Universitaires

ICOM : Comité International des Musées

CNAM : Conservatoire National des Arts et Métiers

USEK : Université Saint-Esprit de Kaslik, Liban

MAU : Musée Archéologique Universitaire

\section{Bibliographie}

BAL Mieke, 1996 : Double Exposures, Londres, Routledge.

BULLETIN DE L'USEK, s. d. : $n^{\circ}$ 74, septembre, p. 92. Disponible sur https://www.usek.edu.lb/museum (consulté le 11 juin 2019).

ChAUmier Serge, 2003 : Des musées en quête d'identité: Écomusée versus technomusée, Paris, L'Harmattan.

Conservatoire national des Arts et Métiers, s. d.: Cnam [en ligne]. Disponible sur http://www.cnam.fr/ (consulté le 11 juin 2019).

CousIN Jean, 1980 : L'espace vivant: Introduction à l'espace architectural premier, Paris, du Moniteur.

DAVALLON Jean, 1999 : L'exposition à l'œuvre: Stratégies de communication et médiation symbolique, Paris, L'Harmattan.

GHARSSAlLAH-Hizem Soumaya, 2008 : Le rôle de l'espace dans le musée et dans l'exposition : Analyse du processus communicationnel et signifiant, thèse de doctorat en muséologie, médiation, patrimoine, Montréal, Université du Québec à Montréal, Université d'Avignon et des pays de Vaucluse.

Giebelhausen Michaela, 2006 : The architecture is the museum, New Museum theory and practice, an introduction, Oxford, Blackwell Publishing.

LETESSON Quentin, 2009 : Du Phénotype au génotype: Analyse de la syntaxe spatiale en architecture minoenne, Louvain, Presses universitaires de Louvain.

NiCOLAS Gilbert, 2020 : Entre un espace muséologique et muséographique. Étude de cas : le Musée Archéologique de I'Université Saint-Esprit - Kaslik, mémoire de master en muséologie, Beirut, Université Libanaise. 
Soubiran Sébastien, Lourenço Marta C., WittJe Roland, Talas Sofia, \& Bremer Thomas, 2009 : «Initiatives européennes et patrimoine universitaire », La Lettre de l'OCIM, n 123, mai-juin, p. 5-14. Disponible sur https://journals.openedition.org/ocim/229 (consulté le 6 décembre 2019).

StAWIARSKI Marcin, 2010: "Rien que des seuils: réflexion sur l'esthétique de l'interstice", Conserverie mémorielle, $\mathrm{n}^{\circ} 7$, avril. Disponible sur https://journals.openedition.org/cm/451\#notes (consulté le 6 décembre 2019).

\section{Notice biographique}

Gilbert Nicolas est un architecte libanais qui a fait ces études en architecture à l'Université Libanaise. Il est titulaire d'un master de recherche en territoire paysage et architecture et d'un master en art et archéologie section muséologie. Co-fondateur de l'atelier d'architecture NCA studios, et enseignant de l'histoire de l'art et de l'architecture à l'école technique du collège central Jounieh - Liban.

Contact : gilbertnicolas@ncastudios.com 